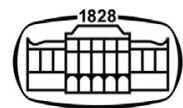

AKADÉMIAI KIADÓ

European Journal of Microbiology and Immunology

$11(2021) 1,1-9$

DOl:

10.1556/1886.2021.00001

(c) 2021 The Author(s)

\section{A literature survey on antimicrobial and immune-modulatory effects of butyrate revealing non-antibiotic approaches to tackle bacterial infections}

\author{
KE DU, STEFAN BERESWILL and MARKUS M. HEIMESAAT* $\odot$
}

Institute of Microbiology, Infectious Diseases and Immunology, Gastrointestinal Microbiology Research Group, Charité - Universitätsmedizin Berlin, corporate member of Freie Universität Berlin, Humboldt-Universität zu Berlin, and Berlin Institute of Health, Berlin, Germany

Received: January 25, 2021 - Accepted: February 19, 2021

Published online: March 17, 2021

\begin{abstract}
The excessive prescription of antibiotics has led to an increasing number of antimicrobial resistances, posing a major public health concern. Therefore, the pharmacological research has shifted its focus to the identification of natural compounds that exhibit anti-pathogenic properties without triggering antibiotic resistance. Butyrate has received increasing attention as a promising candidate for the treatment of bacterial infections in the gastrointestinal tract, particularly when antibiotic treatment is contraindicated. This literature survey summarizes recently investigated antibacterial and immunemodulatory effects of butyrate. This survey revealed that butyrate exerts direct antimicrobial effects against distinct strains of Acinetobacter baumannii, Escherichia coli, Bacillus, and Staphylococcus species. In addition, in vitro and in vivo studies confirmed indirect antimicrobial effects of butyrate, which were exhibited via induction of host defensin production as well as by activation of innate and adaptive immune responses. Finally, the synergistic action of butyrate in combination with other antimicrobial compounds results in a striking clearance of bacterial pathogens. In conclusion, butyrate and its derivatives might be considered as promising antibacterial and immune-modulatory agents in order to tackle bacterial infections without antibiotics.
\end{abstract}

\section{KEYWORDS}

Butyrate, butanoic acid, butyrate analogs, antimicrobial effects, immune-modulatory effects, novel antimicrobial therapies, antimicrobial peptides, host defense peptides, multidrug-resistant (MDR) bacteria

\section{INTRODUCTION}

\section{Antibiotic resistance}

The excessive prescription of antibiotics in human and veterinary medicine has led to an increasing number of antimicrobial resistances among common bacterial pathogens, posing as a major public health concern of increasing magnitude [1-3]. Multi-drug resistant (MDR) bacteria are linked to increased morbidity and mortality, but also present a major obstacle in selecting rational antibiotic therapy in severely ill patients [4-6]. Therefore, MDR strains of bacterial pathogens have translated a socioeconomic burden due to the high resource utilization necessary for their management $[1,4,6,7]$. Mechanisms involved in the development of antimicrobial resistance in both Gram-negative and Gram-positive bacteria can be considered as an evolutionary response to antibiotics and might result in the following events: (i) chemical alteration or destruction of the antibiotic molecule, (ii) decreased antibiotic penetration and/or overexpression of efflux-pumps, (iii) modifications of antibiotic target sites by mutations leading to enzymatic alterations or loss of antibiotic binding, and (iv) 
global metabolic adaptions to cope with environmental stressors [1, 5]. In 2018, the World Health Organization (WHO) has recognized the emergence of antimicrobial resistance and published a list of $12 \mathrm{MDR}$ bacteria which should obtain the highest global awareness and priority of action [8]. Most critical MDR species include Acinetobacter baumannii (carbapenem-resistant), Pseudomonas aeruginosa (carbapenem-resistant) and members of the Enterobacteriaceae (carbapenemase- or extended spectrum betalactamase-producing strains), followed by pathogens with high priority, including vancomycin resistant Enterococcus faecium (VRE), methicillin resistant Staphylococcus aureus (MRSA), vancomycin resistant, and vancomycin intermediate susceptible S. aureus, Helicobacter pylori (resistant to clarithromycin), Neisseria gonorrhoeae (resistant to third generation cephalosporins and fluorochinolones), Campylobacter species (spp.) and Salmonella spp. (both fluoroquinolone resistant). Antibiotic resistances of Streptococcus pneumoniae, Haemophilus influenzae and Shigella spp. to penicillins, macrolides, and fluorochinolones, respectively, receive medium priority [8].

The extensive use of antibiotics in livestock feed for growth promoting purposes comes at costs and has already spread antimicrobial resistance genes through foodborne routes and direct contact, threatening both animal, and human health [2]. Given the inevitability that antibiotic resistant or even MDR bacterial strains will emerge in periods of months to years after an antibiotic enters therapeutic use, the need to develop novel antibiotic independent strategies has become of high importance $[3,7,9]$. In fact, the pharmacological research has shifted its focus to the identification of natural compounds that exhibit anti-pathogenic and anti-inflammatory properties without triggering resistance for future therapeutic and/or preventive applications [9].

\section{Butyrate and host physiology}

Short chain fatty acids (SCFAs) constitute a group of fatty acids with less than six carbons that are generated by anaerobic bacterial fermentation of indigestible dietary fiber, typically reaching concentrations of $50-200 \mathrm{mM}$ in the gut [10-12]. Along with other fermentation derived SCFAs such as acetic acid and propionic acid, butanoic acid is physiologically produced by bacterial communities in the intestinal lumen of the large intestine including the cecum but can also be found in butter and dairy products $[10,12]$. Despite its rancid smell, butyrate, the salt, and anionic part of dissociated butanoic acid, has been established to affect key functions in the immune system, energy metabolism (including insulin resistance) and even the nervous system $[10,12,13]$. Starting from the glycolysis of complex carbohydrates, two molecules of acetyl-CoA are subsequently condensed to acetoacetyl-CoA, which enters a stepwise reduction to butyrate $[11,14,15]$. Phosphotransbutyrylase and butyrate kinase will then convert butyric-CoA to butyrate via the socalled classical pathway. Another route leading to the final formation of butyrate is proceeded by the butyryl-
CoA:acetate CoA-transferase $[11,14,15]$. Despite being the smallest percentage of SCFAs produced, butyrate is the primary energy source in human colonocytes via the $\beta$ oxidation pathway $[10,14]$. Butyrate can efficiently cross the intestinal epithelium by diffusion or via specific transporters including monocarboxylate transporter 1 (MCT1) and sodium-coupled monocarboxylate transporter 1 (SMCT1) [14, 16]. Additionally, MCT4 was recently reported to carry out butyrate-uptake in gut epithelial cells [17], while organic anion transporter 7 (OAT7) was suggested to be a butyratetransporter in hepatocytes [18]. In the gut, butyrate can stimulate $G$ protein-coupled receptors in higher micromolar to millimolar concentrations. So far, GPR41 (also named free fatty acid receptor 3, FFAR3), GPR43 (now FFAR2), GPR109A, and GPR164 (renamed OR51E1) have been identified $[16,19]$.

\section{Immune-modulatory effects of butyrate}

Although the compositions of butyrate-producing (butyrogenic) intestinal bacterial communities vary strongly with age, ethnicity, diet, and gastrointestinal location, a global core community of butyrate producing species in healthy human donors which is composed of Faecalibacterium prausnitzii, Oscillibacter, and Clostridium cluster XIVa was detected using omics data $[20,21]$. Obligate anaerobic $F$. prausnitzii are well-studied acetate-metabolizing and butyrate-producing bacteria, representing about $5 \%$ of the total fecal microbiota in healthy individuals [21-23]. Indeed, a depletion of $F$. prausnitzii has been found in patients suffering from inflammatory bowel diseases like Crohn's disease, for instance [21-23]. Other butyrogenic bacteria include Actinobacteria, Bacteroidetes, Fusobacteria, Proteobacteria, Spirochaetes, and Thermotogae [11, 15]. Decreased butyrate-producing bacterial communities in general are linked to emerging diseases such as type 2 diabetes mellitus, obesity, cardiovascular diseases, ulcerative colitis, and liver cirrhosis [21, 23, 24].

Since the butyrate receptors are expressed ubiquitously in the gut epithelia, in immune cells, and in the enteric nervous system, butyric effects can also affect peripheral organs indirectly by activating hormonal and nervous systems [11, 12]. Moreover, activation of butyrate receptors contributes to the tolerance towards commensal gut bacteria, highlighting the key functional role of butyrate in maintaining intestinal homeostasis and overall health $[13,19]$. In addition to its important position in host energy metabolism, the earliest and most detailed investigated features of butyrate are its immune-modulatory properties $[10,11,13$, $16,25,26]$. According to previous studies, butyrate exhibits histone deacetylase (HDAC) inhibitory abilities and directly affects gene expression by histone hyperacetylation $[11,13]$. Histone acetylation is a central epigenetic controlling mechanism, that modulates the accessibility of the transcriptional machinery by switching between permissive (via acetylation) and repressive (via deacetylation) chromatin structures. In particular, low amounts of physiologically active butyrate tend to stimulate differentiation of intestinal 
Foxp $3^{+}$regulatory $\mathrm{T}$ cells, while higher concentrations have been proven to promote histone $\mathrm{H} 4$ acetylation of $\mathrm{Tbx} 21$ and Interferon- $\gamma$ genes, thereby optimizing the effector function of $\mathrm{CD}^{+}$cytotoxic T lymphocytes and T helper 17 (Th17) cells $[25,26]$. In consequence, butyrate-induced epigenetic regulation is associated with anti-inflammatory and anti-carcinogenic properties [13, 19, 25].

\section{Antimicrobial effects of butyrate}

Regarding previous research results, butyrate has been identified to induce the antimicrobial molecule cathelicidin LL-37 in colorectal epithelial cells in vitro [27, 28]. Cathelicidin LL-37 is an antimicrobial peptide, especially important for host defense at mucosal surfaces [29]. The upregulation of cathelicidin LL-37 transcription via butyrate was linked to an activation of the MEK-ERK pathway [27, 28]. Most importantly, certain pathogens like Shigella have evolved invading mechanisms by down-regulating cathelicidin LL-37 in the colonic epithelium [27]. Another study reported antibacterial effects of sodium butyrate against Corynebacterium pseudotuberculosis in C57BL/6 mice, associated with increased cathelicidin-related antimicrobial peptide expression [30]. In fact, young chicks have been proven to be extremely favorable candidates to study the antibacterial effects of butyrate, since they exhibit low rates of intestinal butyrate production and large growing capacity [31]. Moreover, fat-protected butyrate supplement with extended releasing profile has been widely used in chicken to reduce the cecal Salmonella enterica loads $[32,33]$. In this comprehensive literature survey, we summarize recently investigated antibacterial and immune-modulatory effects of butyrate with a focus on its potential to serve as an antibiotic-independent strategy in the combat of bacterial infections.

\section{METHODS}

\section{Inclusion and exclusion criteria}

The main interest was focused on in vitro and in vivo studies that investigated the antibacterial and immune-modulatory effects of butyrate and butyrate analogs, as well as possible synergistic activities of butyrate with other compounds to identify novel alternatives to antibiotic treatment. Clinical trials have been excluded as well as publications dealing with anti-viral, anti-fungal, and anti-parasitic properties of butyrate.

\section{Search strategy}

Using the MedLine database PubMed we conducted a threestep online literature search from November 28th to December 5th, 2020, by including all publications between 2010 and 2020 that aimed to investigate effects of butyrate directed against bacteria including MDR strains. By utilizing the Boolean logic through the advanced search history, the database was first searched for "butyrate OR butanoic acid" to ensure that synonyms were included. Secondly, the search term "antimicrobial OR antibiotic OR immune-modulatory" was employed, to identify studies that focused on butyratemediated antimicrobial and immune-modulatory effects. Thirdly, by adding the term "antibiotic resistance" to the query we assure that investigations on resistant bacteria were also detected. To narrow down the spectrum of irrelevant results, the bohemian operator "AND" combined all three terms according to the following manner: "(butyrate OR butanoic acid) AND (antimicrobial OR antibiotic OR immune-modulatory) AND (antibiotic resistance)".

In consequence, 87 studies were detected of which 16 qualified for full-text analysis after reading the abstract. By reviewing all 16 articles according to the inclusion and exclusion criteria, six items were withdrawn because they did not focus on the antimicrobial effect of butyrate. One more publication had to be excluded, since it centered on viral diseases. Finally, nine studies could be systematically reviewed in this literature survey.

\section{Data extraction}

All information extracted from the literature search was evaluated with regard to the above-mentioned inclusion and exclusion criteria as the highest priority. Additionally, research design, targeted bacteria, therapeutic concentrations of the applied compound, as well as relevance of control and main findings were taken into consideration.

\section{RESULTS}

\section{Direct antimicrobial effects of butyrate}

The first study assessed the minimal inhibitory concentrations (MICs) of butanoic acid against various Gram-positive and Gram-negative bacteria such as A. baumannii, Bacillus anthracis, Bacillus subtilis, Bacillus megaterium, Escherichia coli, S. aureus, Staphylococcus epidermidis, Staphylococcus intermedius, and Staphylococcus pseudointermedius [34]. Results obtained by applying a viable count assay to quantify bacterial reduction, revealed that butanoic acid inactivated bacteria of all species investigated with a relatively constant MIC between 11 and $21 \mathrm{mmol} / \mathrm{L}$. While B. subtilis and $S$. pseudointermedius were clearly the most susceptible species with $11 \mathrm{mmol} / \mathrm{L}$ sufficient for growth inhibition, the concentrations required for an 8- or even 9-log inactivation remained, however, species dependent. The bactericidal activity of butanoic acid was further explored through visual inspection of cell morphology by using scanning electron microscopy. While A. baumannii, E. coli, and S. pseudointermedius showed no gross morphological changes after butanoic acid treatment, the propidium iodide analysis clearly revealed an increased membrane permeabilization upon exposure. The butanoic acid induced membrane damage was next confirmed in Inductively Coupled Plasma Optical Emission Spectrometry. A. baumannii exhibited a near complete loss of calcium and magnesium after 2 minutes, while the maximum leakage of iron occurred after 6 
minutes exposure to $0.40 \mathrm{~mol} / \mathrm{L}$ of butanoic acid. $E$. coli and S. pseudointermedius experienced a complete loss of magnesium after 4 minutes of exposure. In addition, the presence of butanoic acid led to significant membrane depolarization at lethal concentrations and a rapid cytosolic acidification at sub-inhibitory concentrations [34].

The modifying effects of 2-phenylethyl butyrate (2-PEB) on the antimicrobial activity of the thiomaide drugs ethionamide, isoxyl and thiacetazone were investigated in vitro by determining the growth-inhibitory effects against $M y c o-$ bacterium tuberculosis [35]. Primarily, M. tuberculosis strain H37R and seven drug-resistant isolates were incubated with various ethionamide concentrations in the presence or absence of $0.75 \mathrm{mM}$ 2-PEB. The supplementation of 2-PEB did not only lead to an enhanced antibacterial activity, but also shifted the resistance profiles of MDR M. tuberculosis from resistant to intermediate or from intermediate to susceptible. Since the molecular mechanism of 2-PEB are based on inhibition of ethionamide repressor and enhancement of ethionamide activator, the increased potency of thiomaide drugs is limited to strains with a wild-type ethionamide activator locus only [35].

\section{Stimulation of the innate immune system}

One study elucidated potential antimicrobial effects of butyrate mediated by macrophage differentiation in vitro. To achieve this goal, peripheral blood-derived monocytes were differentiated to macrophages in the presence or absence of butyrate. Both groups of cells were then infected with Gramnegative S. enterica, adherent-invasive E. coli (AIEC), Citrobacter rodentium, and Gram-positive $S$. aureus. The following analyses by gentamicin protection assays revealed that butyrate treated macrophages displayed elevated antibacterial clearance of all the bacterial species, which was maintained even after incubation in butyrate-free media for 24 hours, suggesting a long-term response [36]. In this regard, further analyses for metabolic alterations revealed reduced glycolysis, higher amounts of adenosine monophosphate (AMP) and thereby increased AMP kinase activity with enhanced mechanistic target of rapamycin (mTOR) inhibition. Since mTOR is a well-known regulator for effective autophagy, the authors then confirmed higher amounts of microtubule-associated protein 1A/1B-light chain 3 (LC3) in butyrate treated macrophages, suggesting a mTOR-dependent and LC3-mediated bacterial autophagy [36]. In addition, butyrate treated macrophages were found to produce more reactive oxygen species generated by NADPH oxidase activity before and after S. enterica infection, supporting activation of the pentose phosphate pathway. Considering butyrate's role as an HDAC inhibitor, the treated macrophages were screened for epigenetic changes. Utilizing histone $\mathrm{H} 3$ and $\mathrm{H} 4$ acetylation as an indirect readout, the specificity of HDAC inhibition was examined, revealing that butyrate only acts via HDAC3 inhibition. Next, a single-cell RNA sequencing was performed to identify differentially expressed antibacterial genes, which included up-regulation of effective killing mechanisms against pathogens and down-regulation of classic complement as well as chemokine signaling pathways. Hence, suppressing HDAC3 by butyrate in vitro was sufficient to elevate $S$. enterica clearance without triggering inflammatory cytokine production [36]. Moreover, intracellular staining of calprotectin, a heterodimer of S100A8 and S100A9, was significantly pronounced in a subset of butyrate treated macrophages after 5 days of differentiation. Calprotectin constitutes an antimicrobial protein that exhibits bacteriostatic effects against intracellular pathogens by inducing LC3 or NADPH oxidase, for instance [36].

Addressing the antibacterial activity of butyrate-mediated macrophage differentiation in vivo, C57BL/6 mice were supplemented with butyrate ad libitum for 7 days. After 7 days the ex vivo gentamicin protection assay revealed increased bacterial killings of colonic macrophages compared to the control group. Interestingly, intestinal macrophages of butyrate treated mice displayed elevated antimicrobial activity similar to macrophages treated with butyrate in vitro. Moreover, butyrate treatment of mice reduced bacterial translocation rates after infection with $S$. enterica or $C$. rodentium to peripheral organs including mesenteric lymph nodes, spleen and liver. Although the cecal bacterial load did not differ much from untreated mice, butyrate supplementation lowered the murine inflammatory responses throughout all the bacterial infections under investigation [36].

Given that butyrate is used as a feed additive to protect chicken from bacterial infections, the ability of butyrate to induce host defensive peptides (HDP) was investigated in macrophages, primary monocytes, bone marrow derived cells as well as in jejunal and cecal explants obtained from HD11 chicken [37]. Using different concentrations of sodium butyrate to stimulate the cells at various time points, a real-time PCR (RT-PCR) analysis was performed afterwards to test for avian $\beta$-defensin 9 (AvBD9) expression, a wellknown chicken HDP that is also produced by humans. In macrophages butyrate stimulated AvBD9 expression in a dose-dependent manner. Butyrate at a concentration of 4 $\mathrm{mM}$ resulted in a 5,400-fold induction of gene expression after 24 hours. Similar results were obtained in primary monocytes and in chicken bone marrow derived cells with 200 -fold and 700-fold increases, respectively. Remarkably, several other chicken HDPs, namely cathelicidin B1, AvBD3, AvBD4, AvBD5, AvBD8, AvBD10, and AvBD14, were also triggered by butyrate in a dose-dependent fashion, and similar results were obtained for chicken jejunal and cecal explants [37].

To investigate the HDP induction by butyrate in more detail, 2-day-old chicken were fed with 0.1 or $0.2 \%$ butyrate supplementation prior to their infection with Salmonella enteritidis. Surprisingly, chicks fed with $0.2 \%$ butyrate only demonstrated a 7.5 -fold increase, while $0.1 \%$ butyrate supplementation led to a 22-fold induction of AvBD9. The authors hypothesized that this effect might be explained by the induced growth arrest and apoptotic effects of butyrate when applied in higher concentrations [37]. A further in vivo feeding trial addressed whether oral supplementation of 
butyrate could reduce the cecal bacteria loads after intraesophageal inoculation of chicken with $S$. enteritidis. Supporting earlier findings, $0.1 \%$ butyrate led to a better bacterial reduction in the caecum compared to $0.2 \%$ butyrate treatment. Regarding the dynamics of inflammatory reactions, butyrate only had a minimum impact on the expression of pro-inflammatory cytokines. Given that no effects on Interleukin 1 beta (IL-1 $\beta$ ) or IL-12p40 and only a moderate induction of IL- 8 could be detected after 24 hours, butyrate can be regarded as rather antiinflammatory [37].

To test if butyrate can also induce HDPs in pigs, doseresponse and time-dependent experiments were performed in intestinal porcine enterocyte cells (IPEC-J2) [38]. Expression levels of porcine HDP genes including all so far known cathelicidins (PG1-5, PF1-2, PR-39, PMAP-23, PMAP-36, and PMAP-37) and $\beta$-defensins (pBD1, pBD2, pBD3, pBD4, pBD123, pBD125, pBD129, and pEP2C) were then assessed by RT-PCR. After a 24-hour treatment with 8 $\mathrm{mM}$ sodium butyrate, a remarkable increase of $\mathrm{pBD} 2 \mathrm{mRNA}$ could be found in IPEC-J2 cells. Following exposure to 16 $\mathrm{mM}$ butyrate, also pBD3, pEP2C, and PG1-5 expression could be detected. The butyrate induced HDP expression was next confirmed in porcine 3D4/31lung alveolar macrophages and primary monocytes without triggering a proinflammatory response. In fact, IL- $1 \beta$ remained undetectable throughout butyrate treatment [38].

In a study from 2017, enterohaemorrhagic E. coli (EHEC) was grown in the presence or absence of sodium butyrate, before being challenged with different concentrations of cathelicidin LL-37 [42]. Viable cells were then determined, revealing the surprising observation that the amount of EHEC grown in butyrate was one log order of magnitude higher as compared to the control group. Since previous reports have shown that outer membrane protein $\mathrm{T}(\mathrm{OmpT})$ protects EHEC by degrading cathelicidin LL-37, authors therefore performed PCR analyses, revealing a 3.3-fold higher OmpT gene transcription level in EHEC grown with butyrate. In addition, the locus for enterocyte effacing gene expression, which encodes primary virulence regulatory genes, was activated in response to butyrate. Immunoblotting with FLAG-tagged OmpT gene revealed that the amount of OmpT-FLAG protein in the butyrate group was almost the same as in the control group, implying that the butyrateenhanced cathelicidin LL-37 resistance was rather achieved via increased OmpT secretion [42]. Taking into consideration that Gram-negative bacteria like EHEC are wellknown for their secretion of outer membrane vesicles (OMVs), OMV isolates from EHEC were compared regarding their growth upon co-incubation with and without butyrate. Butyrate did not only lead to a 16 -fold higher amount of OMVs, but also enhanced the quantity of OmpT-loaded OMVs as well as the OmpT activity. In consequence, authors hypothesized that butyrate-induced cathelicidin LL-37 might have trigged resistance against HDPs, suggesting that EHEC evolved the ability to produce more OmpT-loaded OMVs in a co-evolutionary response to the presence of butyrate [42].

\section{Synergistic effects of butyrate in combination with distinct molecules}

The synergistic effects of butyrate with different kinds of natural compounds were assessed in several studies [39-41]. In one study, chicken HD11 and HTC macrophages as well as jejunal explants were treated with $5 \mu \mathrm{M}$ forskolin, a commonly used adenylate cyclase activator, and with $2 \mathrm{mM}$ butyrate for 24 hours [40]. Forskolin has been proven to enhance cathelicidin expression. The following RT-PCR displayed a strong synergistic, 2741-fold increase of AvBD9 expression, which was approximately 3 -fold higher than after treatment with butyrate alone. Notably, a biphasic effect of AvBD9 expression was observed when applying both forskolin and butyrate, since higher concentrations of the combination led to a respectively strong suppression of AvBD9 [40]. To confirm this synergy in vivo, 5-day-old chicken were fed $0.1 \%$ sodium butyrate and $5 \mathrm{mg} / \mathrm{kg}$ Coleus forskohlii extract ad libitum, which contained 10\% forskolin. A significant 630-fold induction of the AvBD9 gene expression was found when the combination was applied. However, higher doses of the C. forskohlii extract resulted in a less pronounced synergistic effect, which is well in line with the obtained in vitro data [40].

Results from another in vitro experiment testing the interactions of butyrate and newly identified HDP-inducing compounds revealed a strong synergy [41]. By applying a stable reporter cell line, a high-throughput screening assay was developed. Natural product and rare natural product libraries were screened for new HDP inducers, revealing 21 compounds. Next, eight compounds were further investigated for AvBD9 induction, with wortmannin, tetrandrine, and datiscetin being the most potent ones. For instance, 4 $\mathrm{mM}$ butyrate and $40 \mu \mathrm{M}$ wortmannin exhibited a 200 - and 250-fold AvBD9 induction, respectively, when applied separately. In combination, however, a dramatic 15,500-fold increase in AvBD9 induction could be detected, reflecting a striking synergy with an additional 60 -fold over each substance alone. In vivo, the combination augmented the antibacterial activity of chicken monocytes after incubation with S. enteritidis, resulting in a nearly complete bacterial growth suppression up to 24 hours post-infection. The combinations butyrate and tetrandrine, as well as butyrate and datiscetin revealed similar results [41].

In another in vitro study chicken HD11 cells and primary monocytes were incubated with three SCFAs, including acetate, propionate and butyrate, separately or in combination for 24 hours, before performing a RT-PCR in order to assess the AvBD9 expression [39]. While application of each fatty acid alone resulted only in a rather subtle AvBD9 induction, the combination of all three SCFAs revealed remarkable synergistic effects as indicated by additional 25- to 50-fold induction in both HD11 cells and primary monocytes. It is noteworthy that a combination of butyrate and propionate as well as of butyrate and acetate did not exhibit any synergistic effects [39]. Furthermore, a HDAC assay revealed that a simultaneous treatment of chicken HD11 cells with acetate, propionate, and butyrate 
resulted in the highest inhibition of HDAC activity with $83 \%$, indicating a great impact of histone deacetylation on the AvBD9-inducing activity [39].

To further analyze the impact on histone deacetylation on the AvBD9-inducing activity in vivo, 4-day-old broilers were supplemented with $0.5 \%$ acetate, $0.2 \%$ propionate, and $0.1 \%$ butyrate in combination, following an inoculation with S. enteritidis. The cecal bacterial loads were dramatically reduced by approximately 7 -fold, supporting the synergistic effects and consistent AvBD9-induction seen in vitro [39].

\section{Further findings}

Butyrate analogs such as benzyl butyrate, glyceryl tributyrate, and trans-cinnamyl butyrate were used to stimulate IPEC-J2 cells, in order to test for their ability to stimulate HDP expression in pigs [38]. While glyceryl tributyrate exhibited a comparable effectiveness to sodium butyrate in triggering all porcine HDPs, benzyl butyrate, and transcinnamyl butyrate acted significantly weaker. Considering that glyceryl tributyrate is odorless, it displays a strong advantage against sodium butyrate when applied in clinical trials [38].

In a murine antibiotic-associated gut dysbiosis model, mice were administered broad-spectrum antibiotics with or without the addition of butyrate before fecal microbiota transplantation [43, 44]. Supplementation with butyrate prevented immune dysfunction and barrier injuries by reducing the secretion of pro-inflammatory cytokines such as tumor necrosis factor alpha $(\mathrm{TNF} \alpha)$ in intestinal macrophages. Butyrate also enabled macrophage activation and directly reprogrammed metabolic behavior by increasing oxidative phosphorylation, further suggesting potential antiinflammatory properties of butyrate. In fact, recolonization after antibiotic-induced changes in the gut microbiota composition was highly associated with colonic $\mathrm{T}$ cell dysfunction, which was significantly reduced by butyrate supplementation even 20 days after recolonization [44]. This is in line with results from another in vitro study, using the Simulator of the Human Intestinal Microbial Ecosystem to compare high-fiber and high-protein diets after challenging with an antibiotic cocktail [45]. Since the high-fiber diet group recovered faster from the antibiotic administration, authors linked this effect to higher butyrate levels due to increased butyrate-producing species like Clostridium cluster XIVa [43-45].

In another study the novel phenolic lipid ferrulic acid esterified with two butyl chains was analyzed to serve as a potential butyrate-prodrug for its antimicrobial properties in vitro [46]. Therefore, Gram-positive (B. subtilis and S. aureus) as well as Gram-negative (E. coli, Pseudomonas aeroginosa, Pseudomonas oleovorians, and Klebsiella spp.) bacterial strains were incubated each with 100 and $150 \mathrm{mg} / \mathrm{L}$ of the synthesized phenolic lipid for 24 hours. Overall, a higher antimicrobial susceptibility was found for the four Gramnegative species tested if compared to Gram-positive bacteria, with $P$. oleovorians being the most susceptible species. In addition, the phenolic lipid exerted moderate antibacterial activities against Gram-positive bacteria in a dose-dependent manner. To test theoretical drug likeness properties in order to predict antimicrobial effects, the Molinspiration software was utilized, which provides physico-chemical data by offering a fragment-based bioactivity prediction. The software revealed better absorption and permeation, thus improving the bioavailability of the compound and posing as a potential prodrug of butanoic acid [46].

\section{DISCUSSION}

\section{Antibacterial activity and immune modulation}

The results from the comprehensive literature survey presented here demonstrate that butyrate exerts multifaceted antibacterial and immune-modulatory effects against Gramnegative and Gram-positive bacteria in vitro and in vivo. Particularly, butyrate imprints cells of the innate immune system via metabolic and epigenetic changes to trigger expression of antimicrobial activities as shown for macrophage differentiation [36]. The elevated bacterial clearance of S. enterica, AIEC, C. rodentium, and S. aureus in vitro [34], as well as the reduced bacterial dissemination of $S$. enterica and C. rodentium in vivo [36], were associated with reduced pro-inflammatory responses, pointing towards butyrate's ability to exhibit antibacterial effects without disrupting intestinal cytokine balance. The high potential of butyrate to enhance immune function was shown to result not only from its direct immune-modulatory properties due to HDAC inhibition, but also from ameliorating $\mathrm{T}$ cell dysfunction following commensal bacterial recolonization after antibiotic-induced gut dysbiosis [43, 44]. Other direct antibacterial actions of butyrate against $A$. baumannii, $B$. anthracis, B. subtilis, B. megaterium, E. coli, S. epidermidis, $S$. intermedius, and $S$. pseudintermedius were linked to membrane damage with depolarization and leakage of intracellular electrolytes [34]. Furthermore, butyrate as well as butyrate analogs were capable of triggering HDPs in vitro and in animal feeding trials in vivo [37-41]. The most distinct results for AvBD9 expression were achieved with $0.1 \%$ butyrate supplementation, whereas higher concentrations even suppressed HDP induction, highlighting the fact that the optimal amount of butyrate must be carefully quantified for each animal species before applying in vivo [37]. Even though most studies reported antibacterial effects in direct or indirect response to butyrate, one study revealed rather contrasting results, given that the presence of butyrate promoted cathelicidin LL-37 resistance through OmpTloaded OMVs in EHEC [42]. Since butyrate is a well-known cathelicidin LL-37 inducer, it seems evident that certain pathogens including EHEC and Shigella have evolved novel invading mechanisms by down-regulating cathelicidin LL37 , thus posing a threat against human HDP barriers [27, 42].

Furthermore, synergistic effects were found for butyrate in combination with other natural HDP-inducing compounds such as forskolin, wortmannin, tetrandrine, and 
datiscetin, revealing a significantly increased AvBD9 induction [40,41]. In addition to the similar synergistic properties regarding AvBD9 expression, an elevated HDAC inhibition could be observed, when butyrate was simultaneously applied with acetate and propionate [38]. Coapplication of 2-PEB with thiomaide drugs such as ethionamide, isoxyl, and thiacetazone was even sufficient enough to alter resistance profiles of $M$. tuberculosis and overcome most of the antibiotic resistant strains [35].

\section{Open questions and areas of future research}

In this review, the antibacterial and immune-modulatory effects of butyrate were evaluated in diverse in vitro and in vivo experiments performed in the past ten years. Considering the limited numbers of studies, distinct conclusions regarding the effects of butyrate should be interpreted with caution. In any case, it is necessary to explore the complexity of heath-beneficial activities exerted by butyrate in welldesigned studies in the future.

The effects of butyrate could be divided in "direct antibacterial effects" and "indirect antimicrobial properties associated with immune modulation". Although only a few in vitro studies revealed direct antibacterial effects of butyrate via cell membrane damage and leakage of electrolytes, promising results were found against Bacillus species, Staphylococcus species and MDR M. tuberculosis, for instance [34]. In most afore-discussed experiments butyrate acted in an indirect manner, by inducing endogenous HDPs or imprinting an antimicrobial program in macrophages and other immune cells [36-41]. HDPs pose as effective agents against pathogens, considering the fact that they are species dependent and exhibit multifaceted killing mechanisms subdivided in heterogeneous groups. Thus, they display a strong advantage against conventional antibiotics, which only affect distinct target molecules within the pathogen. Given that compounds capable of modulating HDP synthesis were initially identified as strategies with low risk of triggering resistance, butyrate associated cathelicidin LL-37 resistance in EHEC and Shigella have been reported in the last years [27, 42]. A plausible explanation for this novel evasion strategy might lie in the co-evolution of the host immune system and the bacterial metabolism [42]. Even though this survey only revealed one in vitro study addressing promising antibacterial effects of butyrate combating drug resistant $M$. tuberculosis [35], possible bactericidal effects of butyrate against other MDR strains should be further investigated.

The capability of butyrate to modulate immune responses to tackle bacterial challenges should be another area of future research. It is noteworthy, that most metabolic changes in immune cells were imprinted via HDAC3 inhibition, suggesting a variety of epigenetic changes effecting metabolic pathways towards long-lasting pro-inflammatory responses [36-41]. Future studies should therefore expand these promising findings regarding butyrate mediated protection against immune cell dysfunction and dysbiosis of the commensal gut microbiota.
In most afore-discussed experiments, butyrate was combined with other natural HDP-inducing compounds or synthetic antibiotics and gained promising results by increasing antibacterial activity in a synergistic manner [3941]. It is of utmost importance to conduct further studies exploring molecular mechanism underlying these synergistic properties, since each combination might be a promising approach to reduce the routine abuse of antibiotics in livestock feed. In this regard, the treatment dose for each animal species must be determined with caution, considering the fact that butyrate is quickly metabolized at insufficient doses and exhibits potential apoptotic effects when applied too excessively [37]. Since butyrate and butanoic acid display a strong unpleasant smell, odorless butyrate analogs and butyrate-prodrugs point towards an attractive alternative when applied in clinical trials $[38,46]$. In this regard, more detailed experimental studies need to be performed in order to find the optimal doses and appropriate way of application for future therapeuticc and/or preventive measures for combating infectious diseases in a butyrate (co-)dependent manner.

\section{Limitations of the literature survey}

Given the rather small number of reviewed publications and heterogenous group of studies with different experimental arrangements, drug concentrations and other confounding variables, conclusions concerning antimicrobial and immune-modulatory effects of butyrate should be carefully drawn. Utilizing the methods outlined in the search strategy the quality assessment of this review was performed as sensitive as possible. However, research mistakes cannot be fully excluded, since data collection and analysis were conducted by a single investigator. Of note, there might be relevant publications that were not evaluated.

\section{Conclusion}

Butyrate and butyrate analogs either alone or in combination with other health-promoting, natural compounds might be considered as promising antibacterial and immunemodulatory agents in order to tackle bacterial infections of the vertebrate host in an antibiotic independent fashion.

Ethical approval: Not applicable (literature survey).

Funding: This work was supported by grants from the German Federal Ministries of Education and Research (BMBF) in frame of the zoonoses research consortium PACCampylobacter (IP7/01KI1725D) and from the Federal Ministry for Economic Affairs and Energy following a resolution of the German National Parliament, Deutscher Bundestag (ZIM, ZF4117908 AJ8) to SB and MMH.

The funders had no role in study design, data collection and analysis, decision to publish or preparation of the manuscript.

Authors' contribution: $\mathrm{KD}$ conceived and designed the survey, wrote the paper. SB provided critical advice in design of 
the survey, edited paper. MMH supervised the survey, cowrote the paper.

Conflict of interest: SB and $\mathrm{MMH}$ are Editorial Board members.

\section{LIST OF ABBREVIATIONS}

\begin{tabular}{|c|c|}
\hline 2-PEB & 2-phenylethyl butyrate \\
\hline AMP & adenosine monophosphate \\
\hline AvBD9 & avian $\beta$-defensin 9 \\
\hline FFAR & free fatty acid receptor \\
\hline GPR & G protein-coupled receptors \\
\hline HDAC & histone deacetylase \\
\hline HDP & host defense peptide \\
\hline IL & Interleukin \\
\hline IPEC-J2 & intestinal porcine enterocyte cells \\
\hline LC3 & 1A/1B-light chain 3 \\
\hline MCT & monocarboxylate transporter \\
\hline MDR & multi-drug resistant \\
\hline MIC & minimal inhibitory concentration \\
\hline mTOR & mechanistic target of rapamycin \\
\hline OAT7 & organic anion transporter 7 \\
\hline OmpT & outer membrane protein $\mathrm{T}$ \\
\hline OMV & outer membrane vesicle \\
\hline RT-PCR & real-time polymerase chain reaction \\
\hline SCFA & short chain fatty acid \\
\hline $\begin{array}{l}\text { SMCT1 } \\
\text { spp: }\end{array}$ & $\begin{array}{l}\text { sodium-coupled monocarboxylate transporter } 1 \\
\text { species }\end{array}$ \\
\hline Th17 cell & $\mathrm{T}$ helper 17 cell \\
\hline $\mathrm{TNF} \alpha$ & tumor necrosis factor alpha \\
\hline WHO & World Health Organization \\
\hline
\end{tabular}

\section{REFERENCES}

1. Munita JM, Arias CA. Mechanisms of antibiotic resistance Microbiol Spectr 2016;4. https://doi.org/10.1128/microbiolspec. VMBF-0016-2015.

2. Xiong W, Wang Y, Sun Y, Ma L, Zeng Q, Jiang X, et al. Antibioticmediated changes in the fecal microbiome of broiler chickens define the incidence of antibiotic resistance genes. Microbiome 2018;6:34. https://doi.org/10.1186/s40168-018-0419-2.

3. Walsh C. Molecular mechanisms that confer antibacterial drug resistance. Nature 2000;406:775-81. https://doi.org/10.1038/ 35021219.

4. Al-Hasan MN, Eckel-Passow JE, Baddour LM. Impact of healthcare-associated acquisition on community-onset Gram-negative bloodstream infection: a population-based study: healthcare-associated Gram-negative BSI. Eur J Clin Microbiol Infect Dis 2012;31: 1163-71. https://doi.org/10.1007/s10096-011-1424-6.

5. Harbottle H, Thakur S, Zhao S, White DG. Genetics of antimicrobial resistance. Anim Biotechnol 2006;17:111-24. https://doi. org/10.1080/10495390600957092.

6. Cerceo E, Deitelzweig SB, Sherman BM, Amin AN. Multidrugresistant gram-negative bacterial infections in the hospital setting: overview, implications for clinical practice, and emerging treatment options. Microb Drug Resist 2016;22:412-31, https://doi.org/10. 1089/mdr.2015.0220.

7. Ciorba V, Odone A, Veronesi L, Pasquarella C, Signorelli C. Antibiotic resistance as a major public health concern: epidemiology and economic impact. Ann Ig 2015;27:562-79. https://doi. org/10.7416/ai.2015.2048.

8. Tacconelli E, Carrara E, Savoldi A, Harbarth S, Mendelson M, Monnet DL, et al. Discovery, research, and development of new antibiotics: the WHO priority list of antibiotic-resistant bacteria and tuberculosis. Lancet Infect Dis 2018;18:318-27. https://oi.org/ 10.1016/s1473-3099(17)30753-3.

9. Hernando-Amado S, Coque TM, Baquero F, Martínez JL. Defining and combating antibiotic resistance from one health and global health perspectives. Nat Microbiol 2019;4:1432-42. https://doi.org/ 10.1038/s41564-019-0503-9.

10. Fu X, Liu Z, Zhu C, Mou H, Kong Q. Nondigestible carbohydrates, butyrate, and butyrate-producing bacteria. Crit Rev Food Sci Nutr 2019;59:S130-52. https://doi.org/10.1080/10408398.2018.1542587.

11. Koh A, De Vadder F, Kovatcheva-Datchary P, Bäckhed F. From dietary fiber to host physiology: short-chain fatty acids as key bacterial metabolites. Cell 2016;165:1332-45. https://doi.org/10. 1016/j.cell.2016.05.041.

12. Stilling RM, van de Wouw M, Clarke G, Stanton C, Dinan TG, Cryan JF. The neuropharmacology of butyrate: the bread and butter of the microbiota-gut-brain axis? Neurochem Int 2016;99:110-32. https://doi.org/10.1016/j.neuint.2016.06.011.

13. Arpaia N, Campbell C, Fan X, Dikiy S, van der Veeken J, deRoos P, et al. Metabolites produced by commensal bacteria promote peripheral regulatory T-cell generation. Nature 2013;504:451-5. https://doi.org/10.1038/nature12726.

14. Astbury SM, Corfe BM. Uptake and metabolism of the short-chain fatty acid butyrate, a critical review of the literature. Curr Drug Metab 2012;13:815-21. https://doi.org/10.2174/138920012800840428.

15. Louis P, Flint HJ. Formation of propionate and butyrate by the human colonic microbiota. Environ Microbiol 2017;19:29-41. https://doi.org/10.1111/1462-2920.13589.

16. Sivaprakasam S, Prasad PD, Singh N. Benefits of short-chain fatty acids and their receptors in inflammation and carcinogenesis. Pharmacol Ther 2016;164:144-51. https://doi.org/10.1016/j. pharmthera.2016.04.007.

17. Kekuda R, Manoharan P, Baseler W, Sundaram U. Monocarboxylate 4 mediated butyrate transport in a rat intestinal epithelial cell line. Dig Dis Sci 2013;58:660-7. https://doi.org/10. 1007/s10620-012-2407-x.

18. Shin HJ, Anzai N, Enomoto A, He X, Kim DK., Endou H, Kanai Y. Novel liver-specific organic anion transporter OAT7 that operates the exchange of sulfate conjugates for short chain fatty acid butyrate. Hepatology 2007;45:1046-55. https://doi.org/10.1002/hep. 21596.

19. Kasubuchi M, Hasegawa S, Hiramatsu T, Ichimura A, Kimura I. Dietary gut microbial metabolites, short-chain fatty acids, and host metabolic regulation. Nutrients 2015;7:2839-49. https://doi.org/10. 3390/nu7042839.

20. Van den Abbeele P, Belzer C, Goossens M, Kleerebezem M, De Vos WM, Thas O, et al. Butyrate-producing Clostridium cluster XIVa species specifically colonize mucins in an in vitro gut model. ISME J 2013;7:949-61. https://doi.org/10.1038/ismej.2012.158. 
21. Vital M, Karch A, Pieper DH. Colonic butyrate-producing communities in humans: an overview using omics data. mSystems 2017; 2. https://doi.org/10.1128/mSystems.00130-17.

22. Martín R, Miquel S, Benevides L, Bridonneau C, Robert V, Hudault $\mathrm{S}$, et al. Functional characterization of novel Faecalibacterium prausnitzii strains isolated from healthy volunteers: a step forward in the use of $F$. prausnitzii as a next-generation probiotic. Front Microbiol 2017;8:1226. https://doi.org/10.3389/fmicb.2017.01226.

23. Foditsch C, Santos TM, Teixeira AG, Pereira RV, Dias JM, et al. Isolation and characterization of Faecalibacterium prausnitzii from calves and piglets. PLoS One 2014;9:e116465. https://doi.org/10. 1371/journal.pone.0116465.

24. Bodogai M, O'Connell J, Kim K, Kim Y, Moritoh K, Chen C, et al. Commensal bacteria contribute to insulin resistance in aging by activating innate B1a cells. Sci Transl Med 2018;10. https://oi.org/ 10.1126/scitranslmed.aat4271.

25. Furusawa Y, Obata Y, Fukuda S, Endo TA, Nakato G, Takahashi D, et al. Commensal microbe-derived butyrate induces the differentiation of colonic regulatory T cells. Nature 2013;504:446-50. https:// doi.org/10.1038/nature12721.

26. Luu M, Weigand K, Wedi F, Breidenbend C, Leister H, Pautz S, et al. Regulation of the effector function of $\mathrm{CD} 8(+) \mathrm{T}$ cells by gut microbiota-derived metabolite butyrate. Sci Rep 2018;8:14430. https://doi.org/10.1038/s41598-018-32860-x.

27. Schauber J, Svanholm C, Termén S, Iffland K, Menzel T, Scheppach $\mathrm{W}$, et al. Expression of the cathelicidin LL-37 is modulated by short chain fatty acids in colonocytes: relevance of signalling pathways. Gut 2003;52:735-41. https://doi.org/10.1136/gut.52.5.735.

28. Schauber J, Iffland K, Frisch S, Kudlich T, Schmausser B, Eck M, et al. Histone-deacetylase inhibitors induce the cathelicidin LL-37 in gastrointestinal cells. Mol Immunol 2004;41:847-54. https://doi. org/10.1016/j.molimm.2004.05.005.

29. Bucki R, Leszczyńska K, Namiot A, Sokołowski W. Cathelicidin LL37: a multitask antimicrobial peptide. Arch Immunol Ther Exp (Warsz) 2010;58:15-25. https://doi.org/10.1007/s00005-009-0057-2.

30. Zhou Z, Yang H, Li H, Li X, Li X, Wu B, et al. Sodium butyrate ameliorates Corynebacterium pseudotuberculosis infection in RAW264.7 macrophages and C57BL/6 mice. Microb Pathog 2019; 131:144-9. https://doi.org/10.1016/j.micpath.2019.04.008.

31. Mátis G, Neogrády Z, Csikó G, Kulcsár A, Kenéz A, Huber K. Effects of orally applied butyrate bolus on histone acetylation and cytochrome P450 enzyme activity in the liver of chicken - a randomized controlled trial. Nutr Metab (Lond) 2013;10:12. https:// doi.org/10.1186/1743-7075-10-12.

32. Bedford A, Gong J. Implications of butyrate and its derivatives for gut health and animal production. Anim Nutr 2018;4:151-9. https://doi.org/10.1016/j.aninu.2017.08.010.

33. Fernández-Rubio C, Ordóñez C, Abad-González J, Garcia-Gallego A, Honrubia MP, Mallo JJ, et al. Butyric acid-based feed additives help protect broiler chickens from Salmonella Enteritidis infection. Poult Sci 2009;88:943-8. https://doi.org/10.3382/ps.2008-00484.

34. Kennedy GM, Min MY, Fitzgerald JF, Nguyen MT, Schultz SL, Crum MT, et al. Inactivation of the bacterial pathogens Staphylococcus pseudintermedius and Acinetobacter baumannii by butanoic acid. J Appl Microbiol 2019;126:752-63. https://doi.org/10.1111/jam.14180. 35. Grau T, Selchow P, Tigges M, Burri R, Gitzinger M, Böttger EC, et al. Phenylethyl butyrate enhances the potency of second-line drugs against clinical isolates of Mycobacterium tuberculosis. Antimicrob Agents Chemother 2012;56:1142-5. https://doi.org/10. 1128/aac.05649-11.

36. Schulthess J, Pandey S, Capitani M, Rue-Albrecht KC, Arnold I, Franchini F, et al. The short chain fatty acid butyrate imprints an antimicrobial program in macrophages. Immunity 2019;50:432-45. e437. https://doi.org/10.1016/j.immuni.2018.12.018.

37. Sunkara LT, Achanta M, Schreiber NB, Bommineni YR, Dai G, Jiang $\mathrm{W}$, et al. Butyrate enhances disease resistance of chickens by inducing antimicrobial host defense peptide gene expression. PLoS One 2011;6:e27225. https://doi.org/10.1371/journal.pone.0027225.

38. Zeng X, Sunkara LT, Jiang W, Bible M, Carter S, Ma X, et al. Induction of porcine host defense peptide gene expression by shortchain fatty acids and their analogs. PLoS One 2013;8:e72922. https://doi.org/10.1371/journal.pone.0072922.

39. Sunkara LT, Jiang W, Zhang G. Modulation of antimicrobial host defense peptide gene expression by free fatty acids. PLoS One 2012; 7:e49558, https://doi.org/10.1371/journal.pone.0049558.

40. Sunkara LT, Zeng X, Curtis AR, Zhang G. Cyclic AMP synergizes with butyrate in promoting $\beta$-defensin 9 expression in chickens. Mol Immunol 2014;57:171-80, https://doi.org/10.1016/j.molimm. 2013.09.003.

41. Lyu W, Deng Z, Sunkara LT, Becker S, Robinson K, Matts R, et al. High throughput screening for natural host defense peptideinducing compounds as novel alternatives to antibiotics. Front Cel Infect Microbiol 2018;8:191. https://doi.org/10.3389/fcimb.2018. 00191.

42. Urashima A, Sanou A, Yen H, Tobe T. Enterohaemorrhagic Escherichia coli produces outer membrane vesicles as an active defence system against antimicrobial peptide LL-37. Cell Microbiol 2017;19. https://doi.org/10.1111/cmi.12758.

43. Duan H, Yu L, Tian F, Zhai Q, Fan L, Chen W. Antibiotic-induced gut dysbiosis and barrier disruption and the potential protective strategies. Crit Rev Food Sci Nutr 2020:1-26, https://doi.org/10. 1080/10408398.2020.1843396.

44. Scott NA, Andrusaite A, Andersen P, Lawson M, Alcon-Giner C, Leclaire $\mathrm{C}$, et al. Antibiotics induce sustained dysregulation of intestinal $\mathrm{T}$ cell immunity by perturbing macrophage homeostasis. Sci Transl Med 2018;10. https://doi.org/10.1126/scitranslmed. aao4755.

45. Marzorati M, Vilchez-Vargas R, Bussche JV, Truchado P, Jauregui R, El Hage RA, et al. High-fiber and high-protein diets shape different gut microbial communities, which ecologically behave similarly under stress conditions, as shown in a gastrointestinal simulator. Mol Nutr Food Res 2017;61. https://doi.org/10.1002/mnfr.201600150.

46. Kaki SS, Kunduru KR, Kanjilal S, Narayana Prasad RB. Synthesis and characterization of a novel phenolic lipid for use as potential lipophilic antioxidant and as a prodrug of butyric acid. J Oleo Sci 2015;64:845-52. https://doi.org/10.5650/jos.ess15035.

\footnotetext{
Open Access. This is an open-access article distributed under the terms of the Creative Commons Attribution-NonCommercial 4.0 International License (https:// creativecommons.org/licenses/by-nc/4.0/), which permits unrestricted use, distribution, and reproduction in any medium for non-commercial purposes, provided the original author and source are credited, a link to the CC License is provided, and changes - if any - are indicated.
} 